\title{
Simulation of Phase Change Material Melting Process in Presence of Nanofluid as an Auxiliary Fluid
}

\author{
Alireza Khademi ${ }^{1}$, Arash Mousemi ${ }^{2}$, Ali Parcheforosh ${ }^{2}$, Mohammad Behshad Shafii ${ }^{2}$, Giancarlo \\ Sorrentino $^{3}$ \\ ${ }^{1}$ Department of Aerospace Engineering, Sharif University of Technology \\ Azadi Ave, Tehran, Iran \\ khademi_alireza@ae.sharif.edu \\ ${ }^{2}$ Department of Mechanical Engineering, Sharif University of Technology \\ Azadi Ave, Tehran, Iran \\ arashmousemi@gmail.com; aliparcheforosh@gmail.com; behshad@ @ sharif.edu \\ ${ }^{3}$ Department of Chemical, Material and Production Engineering, University "Federico II" of Naples \\ Piazzale V. Tecchio, Naples, Italy \\ g.sorrentino@unina.it
}

\begin{abstract}
In this paper, the impact of using auxiliary fluid augmented with nanoparticles on the Phase Change Material (PCM) in a rectangular enclosure is investigated numerically to characterize the melting time and procedure. Considering the properties of the PCMs, Oleic acid (OA) is taken as the PCM, which has the melting point of $13.5^{\circ} \mathrm{C}$. Furthermore, having a higher density, water is exploited as an auxiliary fluid, which is enhanced with a specific volumetric fraction of $\mathrm{CuO}$. It should be mentioned that one advantage of chosen $\mathrm{OA}$ and the nanofluid is that they are separable at the end of the cycle and can be used again because they are insoluble to each other. In this investigation, the chosen nanofluid is on the top of the PCM and is heated from a constant and uniform temperature from its sidewall. At the first time intervals of the melting process and considering that the melted OA has a lower density compared to nanofluid, displacement of fluids occurs, which means the density difference and buoyancy force can influence the melting rate. Shown in the results, the enhancement of water with $\mathrm{CuO}$ nanoparticles can boost the charging process of $\mathrm{OA}$ and reduce its melting time relative to when there is no nanoparticle.
\end{abstract}

Keywords: PCM, Melting process, Auxiliary fluid, Nanoparticles, CFD, Natural convection.

\section{Introduction}

Allowing the decoupling of supply and demand of energy in many cases, Phase Change Materials (PCMs) play a key role in building thermal systems by ameliorating its cost, and they can be used either as a heat source or heat sink. Thermal energy can be stored through sensible heat or latent heat. However, using latent heat, thermal energy can be stored in higher density, and a narrower temperature change of PCM compares to the sensible heat thermal energy storage. Hence, PCMs can be exploited with a considerable number of applications. In this regard, air heating applications [1], air cooling applications in buildings [2], solar energy usage [3], and automotive ones [4] are some instances for a variety cooperation of PCMs in many different fields of tasks.

Various PCMs can be used in extensive temperature ranges which are including the following types in terms of their origin: organic, inorganic, and eutectic [5]. The organic ones can be arranged into paraffin and non-paraffin which are the most common types and unlikely to paraffin; each of them has unique characteristics compared to the others. Moreover, needing an efficient cycle for charging and discharging of PCMs, choosing a PCM with a specific melting point between peak temperature of the application and the minimum temperature of it is extraordinarily vital. Choosing a suitable PCM with this property, melting can occur within the peak temperature period, and solidification can happen when there is minimum demand, or the temperature is lower than the solidification point, which means there is no need for an extra heat exchanger for solidification of the selected PCM which results to higher overall efficiency. Another major factor that can have a massive impact on the study is the geometry of the container as it can change the temperature distribution, affecting 
natural convection of the melted PCM and other related heat transfer qualities. In this case, many studies have been executed by different shapes of containers like spherical, rectangular and cylindrical containers.

Countless studies have been performed in order to enhance the PCMs efficiencies. Sheikholeslami et al. [6] experimentally studied copper oxide nanoparticle to enhance PCM with V shape fins in an enclosure, and they concluded that adding the nanoparticle to pure water can speed up the solidification and also increasing fin length can increase heat penetration depth. Wang et al. [7] numerically investigated the solidification of a device containing multiple PCMs and they conducted that the discharge procedure will expedite in comparison to a single one. Furthermore, Chiu et al. [8] compared and analysed the performance of a system one time with single PCM and latter with a cascade of multiple PCMs, and they revealed that thermal performance using cascade PCMs can be improved by $10-40 \%$ when it comes to the rate of charge or discharge. Besides, they showed that power fluctuation in multi-PCM mode is lower than single-PCM mode.

In the present study, a rectangular storage contains the OA as PCM in bottom and water on the top, which is augmented with $\mathrm{CuO}$ nanoparticle, is heated from one side wall imposing a constant and uniform temperature boundary condition. The nanofluid is on the top of the PCM, so when the melting process starts, the displacement of nanofluid and the melted PCM, which is due to the density difference of them, improves the melting process. Although the charging procedure can thoroughly benefit from performing the mentioned method, this activity has not been investigated in past research works.

\section{Problem Description and Numerical Implementation}

The melted portion of the PCM is considered incompressible and Newtonian. Moreover, the density difference between solid and melted PCM is neglected due to the Boussinesq approximation except where it is multiplied by gravity acceleration. Also, it is presumably to take liquid PCM and nanoparticles in thermodynamic equilibrium and have zero velocity relative to each other. Lastly, numerical method is exploited to simulate the melting of PCM which is placed under $4 \% \mathrm{CuO}$-water nanofluid.

Table 1: Thermophysical properties of oleic acid $[9,10]$, water and nanoparticles [11].

\begin{tabular}{|c|c|c|c|c|c|}
\hline Case & $\begin{array}{c}\rho \\
\left(\mathrm{kg} / \mathrm{m}^{3}\right)\end{array}$ & $\begin{array}{c}\mathrm{C}_{\mathrm{p}} \\
(\mathrm{kJ} / \mathrm{kg} . \mathrm{K})\end{array}$ & $\begin{array}{c}\mathrm{k} \\
(\mathrm{W} / \mathrm{m} . \mathrm{K})\end{array}$ & $\begin{array}{c}\mathrm{d}_{\mathrm{p}} \\
(\mathrm{nm})\end{array}$ & $\begin{array}{c}\text { Viscosity } \\
(\mathrm{kg} / \mathrm{m} . \mathrm{s})\end{array}$ \\
\hline Water & 997.1 & 4.179 & 0.613 & --- & 0.0012692 \\
\hline CuO & 6500 & 0.54 & 18 & 45 & -- \\
\hline Oleic Acid & 840 & 2.2 & 0.2 & --- & 0.03707 \\
\hline
\end{tabular}

According to the mentioned above assumptions, the governing equations of the system can be considered as follows[12]:

$$
\begin{gathered}
\frac{\partial \rho}{\partial t}=-\nabla \cdot(\rho u) \\
\rho\left(\frac{\partial}{\partial t}+u \cdot \nabla\right) u=-\nabla P-\rho g\left[1-\propto\left(T-T_{r e f}\right)\right]+\mu \nabla^{2} u-\frac{C\left(1-f_{l}-f_{n}\right)^{2}}{\delta+\left(f_{l}+f_{n}\right)^{3}} u \\
\rho\left(\frac{\partial}{\partial t}+u \cdot \nabla\right)\left(\left(1-f_{l}-f_{n}\right) C_{s}+f_{l} C_{l}+f_{n} C_{n}\right) T \\
=\nabla\left(\left(1-f_{l}-f_{n}\right) \lambda_{s}+f_{l} \lambda_{l}+f_{n} \lambda_{n}\right) \nabla T-\rho \Delta h \frac{\partial\left(f_{l}\right)}{\partial t}
\end{gathered}
$$




$$
f_{l}=\frac{\Delta H}{\rho \Delta h}\left\{\begin{array}{lr}
0 & \text { if } T \leq T_{s} \\
1 & \text { if } T \geq T_{s} \\
\frac{T-T_{s}}{T_{l}-T_{s}} & \text { if } T_{s}<T<T_{l}
\end{array}\right.
$$

For the above equations, $P, u$ and $T$ represents pressure, velocity and temperature, $\rho$ and $C$ are density and Darcy coefficient which is equal to $1.6^{*} 10^{6} \mathrm{Kg} . \mathrm{m}^{-3}$, respectively. $\delta \ll 1$ is a positive number to avoid dividing by zero which doesn't have any physical meaning in the equation and $\propto$ is thermal expansion coefficient. In addition, $C_{s}$ and $C_{l}$ are specific heat of solid and liquid phase of the PCM respectively and similarly, $\lambda_{s}$ and $\lambda_{l}$ are thermal conductivities of solid and liquid phase of the PCM while $f_{l}$ represents the liquid fraction in mushy zone of PCM, while $\mathrm{n}$ index shows the mentioned parameters related to nanofluid.

\subsection{Mesh Independency and Numerical Validation}

Mesh independency studies were carried out with three different number of elements all generated by structured sized mesh with 70000, 86000 and 102000 cells. Considering that average temperature difference is less than $0.01^{\circ} \mathrm{C}$ by increasing mesh elements from 86000 to 102000 , the mesh number of 86000 is chosen as optimum and final mesh for further solutions.

\subsection{Boundary Conditions}

The no-slip boundary condition is enforced on the walls. Also, Constant and uniform temperature distribution equal to $60^{\circ} \mathrm{C}$ is exploited on the right wall of the system as another boundary condition. However, other walls are insulated.

\section{Results}

The process of OA melting in the presence of nanofluid in different times is illustrated in Fig.1 in which right wall have a constant and uniform temperature boundary condition of $60^{\circ} \mathrm{C}$. At the beginning, melting just occur in the right side because of temperature gradient between OA and wall. Considering that the nanofluid is denser than OA, it replaces with OA during melting process. In this case, melted OA raisin from near the hot wall continually to the point that nanofluid completely cover the right side of OA. However, after nanofluid totally covers the right side, OA will melt from its right side and its particles will raise to the top of the container. Being relatively denser than OA, after a period of time nanofluid will descend in the container causing a faster melting process by melting the deeper parts of OA.

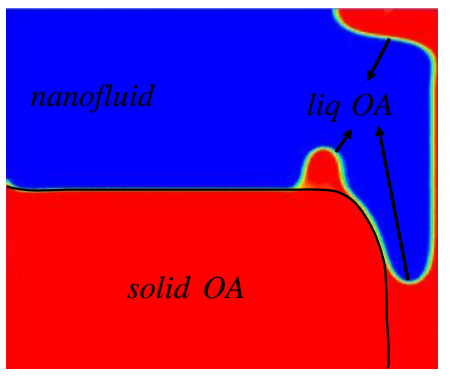

Time $=4 \mathrm{~min}$

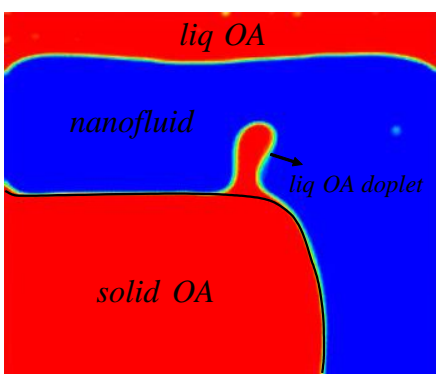

Time $=8$ min

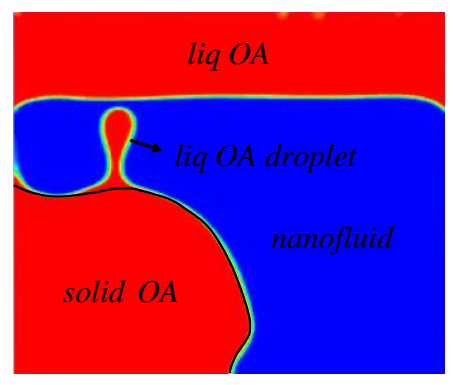

Time $=12 \mathrm{~min}$

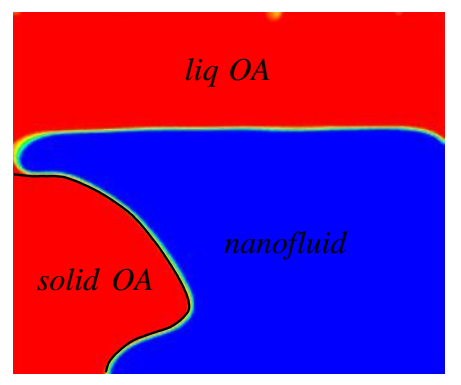

Time $=16 \mathrm{~min}$

Fig. 1: The OA's melting process in the presence of nanofluid at different times.

With a comparison of the presented result with the simulations of Khademi et al. $[9,10]$ in which OA is melted in the presence of pure water in 29 minutes, it can be conducted that rate of charging can be accelerated when water is augmented with nanoparticles which means charging time can be reduced impressively and in this case by 4 minutes. Additionally, 
exploited with nanoparticles, thermal properties of the water are enhanced and consequently more thermal energy can be stored in a shorter time. Thus, the reduction in time is due to adding nano-particles and can be change proportionally by changing volumetric fraction of nanoparticles.

\section{Conclusion}

In this study, melting process of $\mathrm{OA}$ in a rectangular container with the presence of $\mathrm{CuO}$ nanoparticles enhanced water as an auxiliary fluid is numerically investigated. Comparing presented results for pure water and nanofluid, using augmented water with $4 \%$ volumetric fraction of $\mathrm{CuO}$ can positively impact on the melting rate by reducing its time. This reduction in charging time can increase energy storage rate in any cycle as a direct consequence. In other words, by decreasing melting time, more energy can be stored in the system in a specific amount of time. Moreover, the system can be used as a cycle because OA and nanofluid cannot be dissolved in each other and they have different solidification points, which means at the end of the charging process system can be initialized and used for more cycles.

\section{References}

[1] S. Esakkimuthu, A. H. Hassabou, C. Palaniappan, M. Spinnler, J. Blumenberg, and R. Velraj, "Experimental investigation on phase change material based thermal storage system for solar air heating applications," Solar Energy, vol. 88, pp. 144-153, 2013.

[2] F. Souayfane, F. Fardoun, and P.-H. Biwole, "Phase change materials (PCM) for cooling applications in buildings: A review," Energy and buildings, vol. 129, pp. 396-431, 2016.

[3] A. Hasan, S. McCormack, M. Huang, and B. Norton, "Energy and cost saving of a photovoltaic-phase change materials (PV-PCM) system through temperature regulation and performance enhancement of photovoltaics," Energies, vol. 7 , no. 3, pp. 1318-1331, 2014.

[4] J. Jaguemont, N. Omar, P. Van den Bossche, and J. Mierlo, "Phase-change materials (PCM) for automotive applications: A review," Applied Thermal Engineering, vol. 132, pp. 308-320, 2018.

[5] A. Sharma, V. V. Tyagi, C. Chen, and D. Buddhi, "Review on thermal energy storage with phase change materials and applications," Renewable and Sustainable energy reviews, vol. 13, no. 2, pp. 318-345, 2009.

[6] M. Sheikholeslami, R.-u. Haq, A. Shafee, and Z. Li, "Heat transfer behavior of nanoparticle enhanced PCM solidification through an enclosure with V shaped fins," International Journal of Heat and Mass Transfer, vol. 130, pp. 1322-1342, 2019.

[7] P. Wang, D. Li, Y. Huang, X. Zheng, Y. Wang, Z. Peng, and Y. Ding, "Numerical study of solidification in a plate heat exchange device with a zigzag configuration containing multiple phase-change-materials," Energies, vol. 9, no. 6, p. 394, 2016.

[8] J. N. Chiu and V. Martin, "Multistage latent heat cold thermal energy storage design analysis," Applied energy, vol. 112, pp. 1438-1445, 2013.

[9] A. Khademi, M. Darbandi, M. Behshad Shafii, and G. Schneider, "Numerical Simulation of Phase Change Materials to Predict the Energy Storage Process Accurately," in AIAA Propulsion and Energy 2019 Forum, 2019, p. 4225.

[10] A. Khademi, M. Darbandi, and G. E. Schneider, "Numerical Study to Optimize the Melting Process of Phase Change Material Coupled with Extra Fluid," in AIAA Scitech 2020 Forum, 2020, p. 1932.

[11] M. Sheikholeslami and M. Sadoughi, "Simulation of CuO-water nanofluid heat transfer enhancement in presence of melting surface," International Journal of Heat and Mass Transfer, vol. 116, pp. 909-919, 2018.

[12] S. Madruga and J. Curbelo, "Dynamic of plumes and scaling during the melting of a Phase Change Material heated from below," International Journal of Heat and Mass Transfer, vol. 126, pp. 206-220, 2018. 